\title{
A Optimum Model on Urban Public Traffic Line Network for Medium City
}

\author{
Zhao Jing Wang Haiyong \\ School of Electronic and Information Engineering \\ Lanzhou Jiaotong University \\ Lanzhou, China
}

\begin{abstract}
Discuss the urban traffic line network optimization principle, objective and constraints. Presents mathematical expression for distinct optimum objects function form and corresponding constraints. Finally, varied objectives and programming model of public traffic line network optimization is produced, but evaluated and discussed the objective mathematical expression. The public transit optimizing model for medium city is established by analysing the optimizing aim and the restrictive condition.
\end{abstract}

\section{Keywords-Public traffic, Line network, Optimization}

\section{INTRODUCTION}

Medium-sized city's rapid economic development and urbanization, one of the representatives of the urban public transport has failed to catch up with the pace of development level of urban development, so the current urban traffic congestion, the public "ride hard" phenomenon, is still a very prominent issue. Especially as China's urbanization level, improving people's lives, our cities will face more severe traffic challenges, and this situation is difficult in quite a long time to change. There is currently existing transport resources to maximize use efficiency is to ease traffic between supply and demand fundamental way. Since public transport efficient use of transport resources to make by developing public transport, the implementation of transit network optimization as tensions ease urban traffic the necessary road. and the public transportation network Optimization is also a small investment, quick, easy to implement effective measures. Thus the use of modern transportation planning theory and modern computing technology, the existing urban road system and public transport capacity, based on a reasonable public transportation network through the citylayout of the existing public transport capacity to optimize the combination, to maximize the effectiveness of the system's best, has become an important domestic and foreign research scholars. Therefore, this paper draw on research results on the basis of the above, the use of the system of scientific thinking on a number of public transportation network optimization problem.

\section{BASIC KNOWLEDGE OF URBAN PUBLIC TRANSPORTATION NETWORK OPTIMIZATION}

Optimization of urban public transportation network is intended to provide urban residents safe, efficient, economic, convenient and comfortable services; improve the efficiency of bus operations, and promote the development of public transportation, the establishment of good urban traffic environment, and promote land development and urban development, so the city Optimization of transit network must be bus passengers component ( $O D$ matrix) based on the principles of conduct to facilitate urban residents, but also take into account the interests of public transport enterprises and the public transportation network layout of the city traffic.

\section{A. Main Content Of City Line Optimization}

- prediction of traffic demand: According to population surveys and travel forecast urban land use, access to distribution volume of traffic demand matrix.

- line optimization layout: press the "wiring one by one, into a network optimization" method, route optimization in a single basis, considering the overall route network optimization.

- Forecast passenger route network: The previous forecast distribution of urban public transport needs of volume ( $O D$ matrix) assigned to the bus network to be used online to determine the public transit network in each section of the public transport routes and sites up and down passenger traffic volume of passenger flow through the distribution of public transportation network, access to the layout of the program under different route network traffic and site traffic lines, and as an important guide line based on network optimization.

\section{B. Urban Public Transportation}

Network Optimization Principles

- To provide services to more customers, so the trend line must be consistent with the main passenger flow.

- Organization as direct transport, so that the entire service area of total passengers, the minimum transfer times, direct access to the best.

- The shortest distance possible, laid lines, so that the entire service area minimum total travel time of passengers.

- As the distribution of passenger flow on the line, to maximize the vehicle's capacity.

\section{Goal Of City Line Network Optimization}

Intersection optimization model is a typical nonlinear 01programming problem, the objective and constraint functions are complex, consider the optimization 
of urban public transportation network is amulti-objective programming,with mediumsizedcity'spublictransport situati on, identify publictransport Line network optimization objectives are:

- to provide services for more passengers;

- so that all the passengers, the total travel time is smaller, which requires as much as possible to shorten travel distances, reducetransfer times;

- line, the maximum efficiency of nets;

- to ensure proper public transportation network density, ie goodaccessibility;

- ensure that the wire network service area rates, reduce bus blind spot.

D. The constraints of urban public transportation network optimiz-ation

Public transportation network of factors and constraints are more affected by urban passenger traffic demand, road conditions, the station conditions, vehicle conditions, efficiency factors, policies and other factors, constraints, into a single line of the overall constraints and wire mesh constraints. The constraints of a single line in the cloth to consider each line, line line network the constraints of the overall network as a whole after the formation of consideration.

1) Line length limitations: the best line length and average distance related. The average distances that the average traveling distance per passenger trip. In order to reduce the average transfer times, the line should be greater than the average length of the average distance. Line is too short, poor efficiency, increase transfer times; line is too long, the frequency and scheduling of vehicles difficult, staff fatigue. generally requires between approximately $5 \mathrm{~km}$ to $15 \mathrm{~km}$.

2) line of non-linear coefficient restrictions: the length of public transport routes, the terminus of the ratio of straight-line distance between the space, called the line of non-linear coefficient, a non-linear coefficient of the ring line is a major hub points on the line (or The two farthest nodes) between the terms. Lines bend over, driving the inconvenience, but also easy to cause traffic congestion. General line of non-linear coefficient is not greater than 1.4.

3) Section lines (traffic) uniformity coefficient restrictions: uniformity coefficient is the statistical section of time certain traffic routes in operation and the average ratio of road traffic. Uniformity coefficient greater than 1 section of road known as peak passenger flow sections, if necessary, consider opening up within the specified time interval car. General section of the line (traffic) uniformity coefficient not greater than 1.5.

4) Restrictions on the number of passenger's average conversion: the way people travel often transfer from a bus line to another line, and some even transfer several times. The average number of conversions is the total sum divided by the number of transfer passengers, all passengers the number of providers. In general, one-way trip transfer times of urban residents no more than 2 times.

5) Lines overlap coefficient restrictions: overlap factor refers to the passage of the same road sections on the number of bus lines. In the optimization of the number of lines using a complex constraint is the uniformity of the distribution of bus routes, a comprehensive consideration of site parking capacity. Set on a road route number:

$$
N a \leq 3 \sim 5
$$

Where the number of articles for the section of the route.

6) And terminal conditions: Route Optimization station considered in the main constraint is the first and last points of the land.

$$
S_{I} \in S
$$

Where, $S_{I}$ is the starting line of the end; $S$ is from the end of the feasi-ble set.

\section{NONLINEAR MODEL OF MEDIUM URBAN PUBLIC}

\section{TRANSPORTATION NETWORK OPTIMIZATION}

\section{A. Urban Public Transportation Network Optimization Objec-tive Function Expression}

The minimum total travel time of passengers function expression

$$
t=T_{1}+T_{2}+T_{3}+T_{4}+T_{5}
$$

Where $T_{1}$ passenger from the travel point for each station, walk to the appropriate time;

$T_{2}$ is the point from the trip to the station after the appropriate waiting time;

$T_{3}$ is a transit transfer time;

$T_{4}$ is the vehicle to exercise time;

$T_{5}$ is walking off after the arrival time of passengers.

The economic benefits of public transport enterprises (the total daily output of total miles and daily vehicle miles into the ratio of) the best function expression:

$$
\beta=\sum_{\in} L_{s-t} \cdot q_{s-t} / \sum_{s-t} L_{s-t}
$$

$q_{s-t}$ is a transitsnofed $s$ to node $t$ of the traffic.

$K_{s-t}$ is a transit node $s$ to node $t$ of the traffic.

(3) The efficiency of wire mesh (wire mesh of the system benefits the system cost divided by wire mesh) maximum function expression:

\[ \gamma=\sum_{j \in} q_{i j}^{I} \cdot \delta_{i j}^{I} / \sum_{I \in R_{I}} l_{I} \]
$q_{i j}^{I}$ is the route $I$ to site $i^{i, j}{ }^{R}$ from the site's traffic;
$\delta_{i j}^{I}$ is the result of the node $i$ to node $j$ in the line of passenger demand on the distribution ratio $I . l_{I}$ line the length of $I$.

(4) Line network coverage (with public transport in road length / total length of the road network can access) the largest function expression: 


$$
\left[\sum_{L_{s-t} \in L G} L_{s-t}-\sum_{L_{s-t}, L_{s^{\prime}-t} \in L G}\left(L_{s-t} \cap L_{s^{\prime}-t},\right)\right] / \sum_{L_{S-T}} L_{s-t}^{\alpha=}
$$

$L_{s-t}$ line in the bus for the same end of the two adjacent nodes $S$ to $t$ 's distance;

$L_{s^{\prime}-t}$, circular road network is available Node $S^{\prime}$ adjacent to the junction $t^{\prime}$ of the distance.

\section{URban Public Transportation NETWORK OpTIMIZATION MODEL}

Optimal adjustment of public transportation network one of the goals is to consider the interests of passengers, so passengers can easily and quickly travel from this point of view, network density, the bigger the better; but another go-al of optimal adjustment is to consider the business Interests in the limited conditions of construction funds, spend the least money, the most effective trans-formation of public transportation network, apparently reflecting the different interests of these two conflicting objectives. Refer to the "laid one by one, into a network optimization" method to determine alternative routes from the end of ideas and the actual situation in order to minimize passenger travel costs, minimal public transport network construction costs as the objective function, considering the length of bus lines, line carrying capacity, site The maximum allowable number of lines, the total construction cost and other constraints and t-he model. Objective function and the above four constraints corresponding merge to get public transportation network optimization nonlinear model:

$$
\left\{\begin{array}{l}
\operatorname{Min}\left\{T_{1}+T_{2}+T_{3}+T_{4}+T_{5}\right\} \\
\operatorname{Max} \beta \\
\operatorname{Max} \gamma \\
\operatorname{Max} \alpha
\end{array}\right.
$$

Public transportation network planning model is a multiobjective non-linear mathematical programming optimization model is established in the public transportation network is a medium-sized cities to further improve the public transportation network optimization theory, through the medium of urban public transportation network planning Show that the model has strong operational. From the conventional network planning methods compared to the model on the one hand to see the cost savings in the design, improve computational efficiency; the other hand, due to the transportation area assigned to the traffic on the section related to nodes and more fully but also the constraints Design, so to improve the reliability and accuracy of the algorithm and the method is simple and applies to all domestic and medium-sized city.

\section{CONCLUSION}

Optimization of bus network in the medium-sized cities, not only considering the total travel time of passengers and the economic benefits of public transport enterprises, but also takes into accountthe efficiency of nets, so the optimization model to improve the utilization efficiency of existing transport resources, While alleviating the traffic between supply and demand.

The public transportation network in this paper the nonlinear optimization model, not only both the passengers and bus companies about the two interests, but also taking into account the overall efficiency of urban transport system, so the nonlinear optimization model Representation and the use of a certain value. But in real life, due to the complexity of urban transit systems, public transportation network optimization constraints and objective functions need further study and discussion.

\section{REFERENCES}

[1] WANG Wei;. Urban Public Transport System Planning and Management Technology. Beijing: Science Press, 2002, PP:77-79

[2] WANG Zhi-dong;. Public transportation network optimization model. Dalian Railway Institute[J],1997.12, PP:31-34

[3] HAN Yin;. Urban public transportation network on behalf of the PSO algorithmto adjust. China Journal of Highway [J],1997.12, PP:100-105

[4] CHENG Pang-wen;. Design of urban public transit network optimization models and methods. Systems Engineering Theory and Practice [J],1990.7, PP:72-77

[5] ZHAO Zhi-feng;. Urban public transport route network planning. Journal of Shanghai Jiaotong University [J],1988.22, PP:63-67

[6] QIAN Da-lin;. Discrete non-equilibrium transport network optimization model and its solution algorithm. Northern Jiaotong University Journals [J],2001.25, PP:58-61

[7] HU Qi-zhou;. Multi-objective optimization of urban public transportation networkmodeling and solution [J]. Jiangsu University (Natural Science),2003.24, PP:88-90

[8] LIU Wei-hua;. Road layout of the main terminals of quantitative evaluation studies[J]. China Journal of Highway,2003.16, PP:86-89 